\title{
Sleep-wake disturbances in cancer patients: narrative review of literature focusing on improving quality of life outcomes
}

This article was published in the following Dove Press journal:

Nature and Science of Sleep

12 July 2014

Number of times this article has been viewed

\section{Suzanne S Dickerson Laurie M Connors \\ Ameera Fayad Grace E Dean}

School of Nursing, State University of New York, University at Buffalo, NY, USA
Correspondence: Suzanne S Dickerson School of Nursing, State University of New York, University at Buffalo, 30IE Wende Hall, 3435 Main Street, Buffalo, NY 14214, USA

$\mathrm{Tel}+\mathrm{I} 7168293254$

Fax +17168292120

Email sdickers@buffalo.edu
Purpose: Evidence suggests a high prevalence of sleep-wake disturbances in patients with cancer, occurring at diagnosis, during treatment, and continuing to survivorship. Yet associations between sleep-wake disturbances and the impact on quality of life outcomes is less clear. The purpose of this narrative review of the literature is to evaluate sleep-wake disturbances in patients with cancer, to describe the influence of poor sleep on quality of life as an outcome, and to evaluate the evidence to recommend future interventions.

Framework and methods: This review was guided by the Preferred Reporting Items for Systematic Reviews and Meta-Analyses (PRISMA) approach. Four databases (CINAHL, MEDLINE, PsycINFO, and Embase) were searched using terms "cancer OR neoplasm", "sleep, sleep disturbance, sleep disorders or insomnia", and "quality of life"; the search included all years, English language, and peer-reviewed articles on research studies. Studies included measurements of sleep and quality of life in cancer patients at a minimum of two time points and demonstrated relationships between sleep and quality of life. Data were collected on date, patient demographics, cancer type and treatment, timeframe, design, measurement, variables, and results.

Results: This narrative review demonstrates that sleep-wake disturbance is a major problem/ symptom in patients with cancer. Of the 18 studies included, measurement of sleep-related variables included objective and subjective measures; however, direct measurement of the associations between sleep and quality of life was not common. Cognitive behavioral therapy for insomnia and mind-body interventions demonstrated feasibility when implemented into cancer care settings. In addition, the majority of interventions exhibited moderate effectiveness in improving sleep-wake disturbance and quality of life outcomes.

Conclusion: The studies predominantly reported that poor sleep negatively impacts quality of life. The intervention studies included nonpharmacologic interventions such as cognitive behavioral treatment and mind-body and exercise interventions with moderate-to-high levels of evidence for improvement in sleep measures and quality of life.

Keywords: sleep-wake disturbance, quality of life, cancer, cognitive behavioral therapy, mindbody interventions, insomnia

\section{Introduction}

Sleep disturbances are commonly experienced by patients with cancer and are often overlooked due to other diagnostic and treatment-related concerns. ${ }^{1-3}$ Patients describe problems falling asleep (sleep latency), problems staying asleep (awakenings), having restless sleep (quality of perceived sleep), and/or having trouble staying awake during the day (excessive daytime sleepiness). ${ }^{2}$ The issues of sleep-wake disturbances are often combined in symptom clusters, especially with cancer-related fatigue ${ }^{4,5}$ and insomnia, ${ }^{6}$ 
which occur pretreatment, ${ }^{3}$ exacerbate during treatment, and continue through survivorship. During cancer treatment, many patients nap during the day, leading to circadian rhythm problems, which exacerbate sleep problems. ${ }^{2}$ While there is some awareness of sleep-wake disturbances in the context of symptom clusters ${ }^{5,7-9}$ during a patient's cancer trajectory, what is not known is the impact of sleep on the physical, functional, and social aspects of quality of life (QOL). ${ }^{10}$

Adequate sleep is a biologic requirement for healthy physical, cognitive, and psychological functioning; however, acute and chronic sleep deprivation results in negative health consequences such as compromised immunological, cardiovascular, and neuropsychiatric functioning that are exacerbated by cancer diagnosis and treatments, thus influencing the patients' QOL. ${ }^{10}$ QOL is a concept that encompasses how cancer and its treatments affect dimensions of physical (health-related), functional, and mental/psychological/ emotional well-being. QOL has been extensively studied in cancer patients, yet sleep disturbance has often not been included as a main study variable. ${ }^{11}$ Vena et $\mathrm{al}^{10}$ suggest using the two-process model of sleep regulation ${ }^{12}$ to understand sleep-wake mechanisms in cancer patients, which includes a homeostatic process influenced by previous sleep and wake states and a circadian process that determines the propensity for falling asleep and consolidating sleep. This model can then be used to discuss cancer-related factors that influence a patient's risk for developing sleep problems, such as: age and sex; cancer and treatment; lifestyle; and psychological well-being. There is a need to understand current knowledge of sleep-wake disturbances and the influence of these factors on QOL, as well as evidence-based interventions to improve sleep-wake disturbance and QOL. Therefore, the purpose of this paper was to systematically conduct a narrative review of the literature on sleep-wake disturbances and QOL in patients with cancer and to synthesize the evidence of longitudinal descriptive and intervention studies to improve sleep and QOL. The review is organized by study design (longitudinal descriptive and intervention), measurement issues, and discussion, and concludes with suggestions for future practice and research.

\section{Methods}

\section{Framework and search methods}

This narrative review was guided by the Preferred Reporting Items for Systematic Reviews and Meta-Analyses (PRISMA) approach $^{13}$ and Berger et al's methodological review findings. ${ }^{11}$ Four electronic databases (CINHAL, MEDLINE, PsycINFO, and Embase) were searched using the terms "cancer OR neoplasm", "sleep, sleep disturbance, sleep disorders or insomnia, and quality of life"; the search included all years, English language, and peer-reviewed articles of original studies. Figure 1 demonstrates the process of search and review. Data collected included: date, patient demographics, cancer type, treatment/ timeframe, study design, measurement variables, and results. Inclusion criteria were: 1) peer-reviewed original research or secondary analysis of subjects with a cancer diagnosis (omitting review articles and theses), in which the variables were measured at two or more time points; ${ }^{12}$ 2) QOL and sleepwake disturbance measures that demonstrated an association between sleep-wake disturbance and QOL (head and neck cancers were omitted due to potential obstructive airway issues); and 3) studies written in the English language, limited to those involving quantitative methods. ${ }^{11}$

The review process followed procedures from the PRISMA method modified for narrative analysis. After the search was conducted, manuscripts were appraised by a team of four to reach consensus on applicability and quality. The team included: the first author, an academic nurse educator and sleep researcher; the second author, a nurse educator and advanced practice nurse in oncology; the third author, a $\mathrm{PhD}$ student researching sleep in cancer; and the fourth author, a nurse educator and sleep researcher focusing on sleep and fatigue in cancer patients. Any discrepancies were resolved by returning to the original manuscripts and holding discussion to reach consensus. Tables of data were created and validated by consensus. In the synthesis of results and rating of the quality of the interventions, the team assessed the risk of bias, including evaluating each study for recruitment processes, sample size, methods, funding, journal impact, measurement, and study outcomes (effect estimates for intervention studies and confidence intervals for intervention studies when possible). The PRISM checklist was completed to ensure rigor (Table 1).

\section{Results}

The initial search included 682 manuscripts and a further six were identified from hand searches of reference lists. Eighteen studies met the eligibility criteria, including three longitudinal descriptive and 15 intervention studies, all of which were published in peer-reviewed journals. The reviewed studies included 1,340 cancer patients with a mean age of 55.5 years. The majority were female $(88 \%)$ with breast cancer $(66 \%)$ and mixed diagnosis $(33 \%)$.

\section{Study design: longitudinal descriptive}

The three included studies that met the criteria for a descriptive longitudinal design with sleep-wake disturbance 


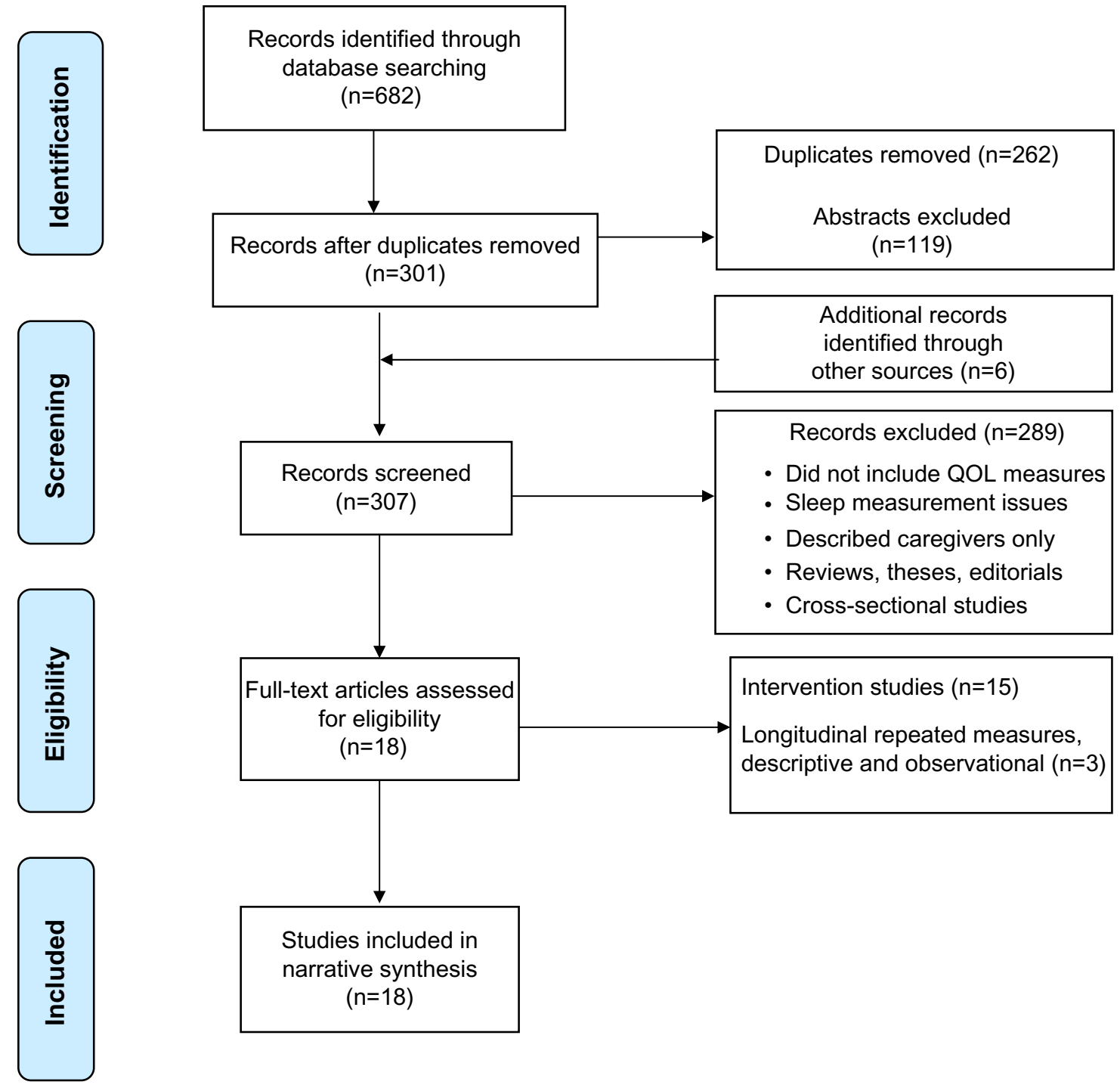

Figure I Flow diagram of the search and review process.

Notes: Reproduced from Moher D, Liberati A, Tetzlaff J, Altman DG, PRISMA Group. Preferred reporting items for systematic reviews and meta-analyses: the PRISMA statement. Ann Intern Med. 2009; I5I:264-269.'

Abbreviation: QOL, quality of life.

and QOL correlations are shown in Table 2. In a recent study by Liu et al,${ }^{14} \mathrm{QOL}$ and subjective sleep quality were the primary variables in 166 patients undergoing chemotherapy for breast cancer. Liu et al found that poor nocturnal sleep and longer naps were associated with poorer physical QOL, and that the mental component of QOL was associated with the subjective reports of poor sleep but not by objective sleep time. The authors concluded that subjective reports logically concur with mental components and that poor sleep and naptime (longer than 30 minutes) may reflect poor sleep habits that contribute to poor sleep quality. ${ }^{14}$ This finding suggests that including sleep hygiene information in the interventions to improve sleep in breast cancer patients may ultimately improve QOL. Similarly, Clevenger et a ${ }^{15}$ studied sleep quality and QOL as primary variables in 173 ovarian cancer patients. Disturbed global sleep was found at all-time points (pre-surgery, at 6 months, and at 1 year) and was associated with depression, pain, sleep medications, and declines in QOL. ${ }^{15}$ Beck et a ${ }^{16}$ studied elderly survivors $(\mathrm{n}=52)$ with an average age of 72.7 years and reported that fatigue and sleep symptoms occurred together. Fifty-eight percent had poor sleep quality, with significant correlations between sleep quality and physical, mental, and functional QOL. ${ }^{16}$ These findings demonstrate the importance of developing interventions to improve sleep quality, especially in older adults with a high rate of comorbidities. 
Table I PRISMA checklist modified for narrative analysis

\begin{tabular}{lll}
\hline Topic & Item & $\begin{array}{l}\text { Page } \\
\text { number(s) }\end{array}$ \\
\hline Title & Identify the report as narrative review. & I \\
\hline Abstract & $\begin{array}{l}\text { Structured abstract including background, } \\
\text { objectives, data sources, study eligibility } \\
\text { criteria, study appraisal and synthesis } \\
\text { method, results, conclusions, and }\end{array}$ & \\
& implications of key findings. &
\end{tabular}

\begin{tabular}{lll}
\hline $\begin{array}{l}\text { Introduction } \\
\text { Rationale }\end{array}$ & $\begin{array}{l}\text { Describe rationale for review in the } \\
\text { context of what is already known. }\end{array}$ \\
Objectives & $\begin{array}{l}\text { Provide explicit statement of questions } \\
\text { being addressed, referring to participants, } \\
\text { interventions, comparisons, outcomes, } \\
\text { and study design. }\end{array}$
\end{tabular}

\begin{tabular}{|c|c|c|}
\hline \multicolumn{3}{|l|}{ Methods } \\
\hline $\begin{array}{l}\text { Research } \\
\text { questions }\end{array}$ & Indicate primary research focus. & 2 \\
\hline $\begin{array}{l}\text { Eligibility } \\
\text { criteria }\end{array}$ & $\begin{array}{l}\text { Specific study characteristics and } \\
\text { report characteristics used as } \\
\text { criteria for eligibility with rationale. }\end{array}$ & 2 \\
\hline $\begin{array}{l}\text { Information } \\
\text { sources }\end{array}$ & $\begin{array}{l}\text { Describe all information sources - } \\
\text { databases, with search terms. }\end{array}$ & 2 \\
\hline Study selection & $\begin{array}{l}\text { State process for selecting studies - } \\
\text { screening for eligibility. }\end{array}$ & 2 \\
\hline $\begin{array}{l}\text { Data collection } \\
\text { process }\end{array}$ & $\begin{array}{l}\text { Describe data extraction from reports } \\
\text { and process of confirming data in tables. }\end{array}$ & 2 \\
\hline Data items & $\begin{array}{l}\text { List all variables for which data is } \\
\text { sought with assumptions and } \\
\text { simplifications. }\end{array}$ & 2 \\
\hline Risk of bias & $\begin{array}{l}\text { Describe methods to assess risk of } \\
\text { bias of individual studies and how } \\
\text { used in synthesis. }\end{array}$ & 2 \\
\hline $\begin{array}{l}\text { Summary } \\
\text { measures }\end{array}$ & $\begin{array}{l}\text { State summary measures in narrative } \\
\text { format. }\end{array}$ & 2 \\
\hline $\begin{array}{l}\text { Synthesis of } \\
\text { results }\end{array}$ & $\begin{array}{l}\text { Describe method of handling data } \\
\text { and combining results of studies. }\end{array}$ & 2 \\
\hline \multicolumn{3}{|l|}{ Results } \\
\hline Study selection & $\begin{array}{l}\text { Number of studies screened, assessed } \\
\text { for eligibility, and included in the review } \\
\text { with reasons for exclusions in a diagram. }\end{array}$ & Figure I \\
\hline $\begin{array}{l}\text { Study } \\
\text { characteristics }\end{array}$ & $\begin{array}{l}\text { For each study, present characteristics for } \\
\text { which data were extracted and rated. }\end{array}$ & $\begin{array}{l}\text { Tables } 2 \\
\text { and } 3\end{array}$ \\
\hline $\begin{array}{l}\text { Synthesis and } \\
\text { rating }\end{array}$ & Present results - narrative. & $2-12$ \\
\hline Discussion & $\begin{array}{l}\text { Summary of evidence, limitations, and } \\
\text { conclusions and implications for future } \\
\text { research. }\end{array}$ & $12-15$ \\
\hline Funding & Describe sources of funding. & NA \\
\hline
\end{tabular}

Notes: Reproduced from Moher D, Liberati A, Tetzlaff J, Altman DG, PRISMA Group. Preferred reporting items for systematic reviews and meta-analyses: the PRISMA statement. Ann Intern Med. 2009;151:264-269.'

Abbreviations: NA, not applicable; PRISMA, Preferred Reporting Items for Systematic Reviews and Meta-Analyses.

These longitudinal studies showed that, when comprehensive sleep measures were implemented in the study design, a clear understanding emerged regarding the influence of poor sleep and sleep behaviors on QOL. Poor sleep quality was moderately associated with poor health-related QOL. Sleep behaviors such as prolonged daytime napping was associated with poor QOL. Such findings suggest the need for interventions that support improvement in sleep and QOL outcomes.

\section{Study design: interventions}

Included in this review are 15 studies that tested interventions to evaluate the feasibility and efficacy of improving sleep and QOL (see Table 3). All studies demonstrated an effect of the intervention on sleep and QOL outcomes, although only one study reported an association between sleep and QOL. Interventions included seven cognitive behavioral interventions and eight mind-body interventions (including three meditation, one yoga, one Qigong, one relaxation, one biofeedback, and one exercise).

\section{Cognitive behavioral therapy for insomnia (CBT-I)}

Seven interventions in this review utilized CBT-I, which improved sleep efficiency as well as QOL, depression, and fatigue. Of these studies, five were randomized controlled trials (RCTs) (including three pilots) and two were repeatedmeasures studies. Davidson et a ${ }^{17}$ tested a group treatment format for 12 patients with insomnia with no control group, utilizing a sleep diary, Sleep Impairment Index, and European Organisation for Research and Treatment of Cancer (EORTC QLQ-C30) to measure sleep disturbance and QOL. There were significant improvements over baseline at weeks 4 and 8 related to sleep efficiency, wake after sleep onset, and total sleep time, with fatigue improving by week 8 ; however, the QOL role-functioning improvement was not significant. With no comparison group and a small sample size, the study was rated lower in quality of evidence as rated by the review team.

Quesnel et al ${ }^{18}$ tested an 8-week CBT-I intervention in a small sample $(\mathrm{n}=10)$ of breast cancer patients with no control group using Pittsburgh Sleep Quality Index (PSQI) and polysomnography to corroborate with sleep diary data for total wake time and sleep efficiency and found moderate-tostrong effects on global QOL, cognitive QOL, and Insomnia Severity Index. The treatment significantly improved sleep, which was sustained over time (6 months), and reduced depression and fatigue. The small sample size and lack of control influenced the quality; however, high-quality sleep measurement identified moderate-to-strong effect sizes in pre-/posttreatment comparisons.

Savard et al ${ }^{19}$ tested CBT-I in an RCT of 57 breast cancer patients with insomnia with a waitlist crossover design and reported that the treatment group showed decreased medication use (15.3\%) and improved sleep efficiency 


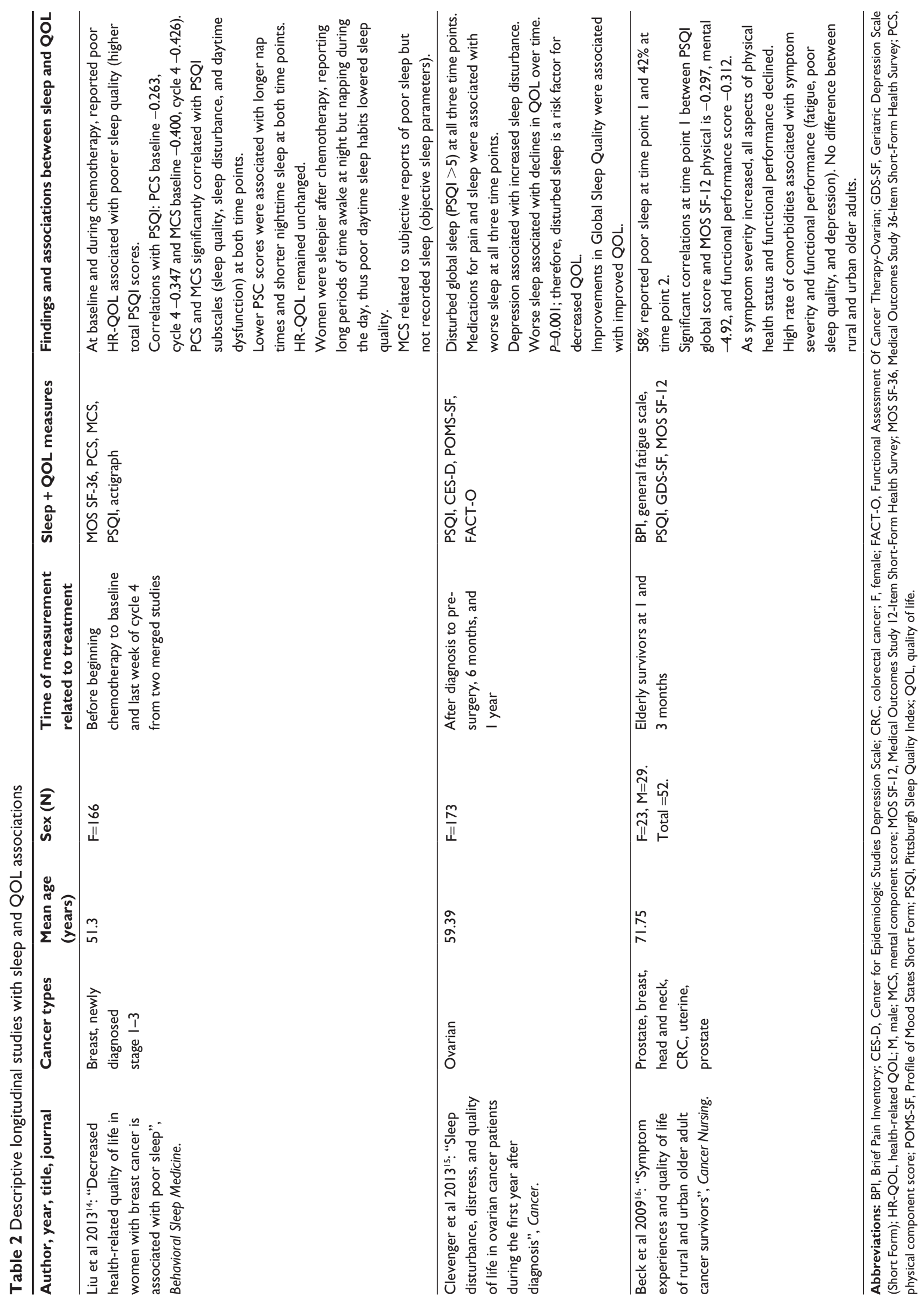


Table 3 Intervention studies $(n=15)$ to improve sleep and QOL

\begin{tabular}{|c|c|c|c|c|}
\hline Author, year & Intervention and design & Sample & Cancer types & Variables/measurement \\
\hline \multicolumn{5}{|c|}{ Cognitive behavioral interventions $(n=7)$} \\
\hline $\begin{array}{l}\text { Davidson et al } \\
2001^{17}\end{array}$ & $\begin{array}{l}\text { Group treatment for insomnia, } \\
\text { six sessions stimulus control, } \\
\text { relaxation, education, and } \\
\text { cognitive restructuring. } \\
\text { Prospective baseline, } 4 \text { and } \\
8 \text { weeks. }\end{array}$ & $\begin{array}{l}\mathrm{N}=12 \text {. } \\
\text { Mean age } 55 \text { years. } \\
\mathrm{F}=\mathrm{II} . \mathrm{M}=\mathrm{I} \text {. } \\
\text { Patients with insomnia. } \\
\text { No control. }\end{array}$ & $\begin{array}{l}\text { Mixed. } \\
\text { Breast, GI, GYN. }\end{array}$ & $\begin{array}{l}\text { Sleep diary, SII, sleep disturbance item } \\
\text { on EORTC QLQ-C30. }\end{array}$ \\
\hline
\end{tabular}

\begin{tabular}{lllll}
\hline Quesnel et al & CBT (8 weeks). & $\mathrm{N}=10$. & Breast. & PSQI, PSG (to corroborate with, sleep \\
$2003^{18}$ & Longitudinal repeated & No control. & Nonmetastatic with & diary for TWT and SE), ISI, QLQ-C30. \\
& measures. & Mean age 54.3 years. & insomnia. &
\end{tabular}

\begin{tabular}{|c|c|c|c|c|}
\hline $\begin{array}{l}\text { Savard et al } \\
2005^{19}\end{array}$ & $\begin{array}{l}\text { CBT. } \\
\text { RCT, waitlist crossover. }\end{array}$ & $\begin{array}{l}\mathrm{N}=57 . \\
\text { Treatment }=27 . \\
\text { Control }=30 . \\
\text { Mean age } 54.8 \text { years. }\end{array}$ & $\begin{array}{l}\text { Breast with insomnia. } \\
\text { Outpatient. }\end{array}$ & $\begin{array}{l}\text { Sleep diary, PSG, ISI, EORTC } \\
\text { QLQ-C30. }\end{array}$ \\
\hline $\begin{array}{l}\text { Dirksen and } \\
\text { Epstein } 2008^{22}\end{array}$ & $\begin{array}{l}\text { CBT insomnia intervention. } \\
\text { RCT. }\end{array}$ & $\begin{array}{l}\mathrm{N}=72 . \\
\text { Treatment }=34 . \\
\text { Control }=38 . \\
\text { Mean age } 58 \text { years. }\end{array}$ & $\begin{array}{l}\text { Breast. } \\
\text { Outpatient. }\end{array}$ & FACT-B, ISI. \\
\hline $\begin{array}{l}\text { Espie et al } \\
2008^{23}\end{array}$ & $\begin{array}{l}\text { CBT for insomnia. } \\
\text { RCT versus usual treatment. }\end{array}$ & $\begin{array}{l}\mathrm{N}=150 . \\
\text { Treatment }=100 . \\
\text { Control }=50 . \\
\mathrm{F}=103 . \\
\mathrm{M}=47 . \\
\text { Mean age } 60.5 \text { years. }\end{array}$ & Breast, prostate. & $\begin{array}{l}\text { PSQI, ESS, FACT-G, Actigraphy, } \\
\text { sleep diary, FSI. }\end{array}$ \\
\hline $\begin{array}{l}\text { Savard et al } \\
20 I^{20}\end{array}$ & $\begin{array}{l}\text { Feasibility study of 6-week } \\
\text { self-help treatment for } \\
\text { insomnia comorbid with } \\
\text { cancer. }\end{array}$ & $\begin{array}{l}\mathrm{N}=1 \mathrm{I} . \\
\text { Mean age } 5 \mathrm{I} . \mathrm{I} \text { years. }\end{array}$ & Breast. & $\begin{array}{l}\text { Sleep diary, pre-/post treatment and } \\
\text { 3-month follow-up, ISI, EORTC } \\
\text { QLQ-C30, DBAS. }\end{array}$ \\
\hline
\end{tabular}

\begin{tabular}{|c|c|c|c|c|}
\hline $\begin{array}{l}\text { Ritterband } \\
\text { et al } 2012^{21}\end{array}$ & $\begin{array}{l}\text { Online CBT for insomnia. } \\
R C T \text {, waitlist control. }\end{array}$ & $\begin{array}{l}\mathrm{N}=28 . \\
\text { Treatment }=14 . \\
\text { Control }=14 . \\
\mathrm{F}=24 . \mathrm{M}=4 . \\
\text { Mean age } 56.7 \text { years. }\end{array}$ & $\begin{array}{l}\text { Breast and other } \\
\text { survivors. }\end{array}$ & ISI, MOS SF-12, sleep diary. \\
\hline \multicolumn{5}{|c|}{ Mind-body interventions $(n=8)$} \\
\hline $\begin{array}{l}\text { Simeit et al } \\
2004^{24} \text { (Germany) }\end{array}$ & $\begin{array}{l}\text { Longitudinal. } \\
\text { Two-group. } \\
\text { Multimodal sleep management. } \\
\text { Two groups: PR and AT. } \\
\text { Non-random. }\end{array}$ & $\begin{array}{l}\mathrm{N}=229 . \\
\mathrm{F}=172 . \mathrm{M}=47 . \\
\mathrm{PR}=80, \mathrm{AT}=7 \mathrm{I} . \\
\text { Control }=78 . \\
\text { Mean age } 58 \text { years. }\end{array}$ & $\begin{array}{l}\text { Patients with } \\
\text { insomnia. } \\
\text { Breast, kidney, } \\
\text { prostate. } \\
6 \text { months } \\
\text { posttreatment. }\end{array}$ & PSQI, EORTC QLQ-C30. \\
\hline $\begin{array}{l}\text { Carlson et al } \\
2004^{25}\end{array}$ & $\begin{array}{l}\text { MBSR. } \\
\text { Pre-/post-mindfulness-based } \\
\text { stress reduction intervention. } \\
\text { No control. }\end{array}$ & $\begin{array}{l}\mathrm{N}=69 . \\
\mathrm{F}=59 . \\
\mathrm{M}=10 . \\
\text { Mean age } 54.5 \text { years. }\end{array}$ & Breast, prostate. & $\begin{array}{l}\text { Health behavior form asks hours }+ \\
\text { quality of sleep (good/poor/adequate), } \\
\text { SOSI, cortisol, DHEAS, melatonin, } \\
\text { EORTC QLQ-C30. }\end{array}$ \\
\hline
\end{tabular}




\begin{tabular}{|c|c|c|}
\hline Effect size $(\mathrm{Cl})$ & Results & Quality score \\
\hline $\begin{array}{l}\text { Pre-/8-week scores. } \\
\text { Awakenings }-0.97(-1.85 \text { to }-0.16) \text {. } \\
\text { WASO }-1.18 \text { ( }-2.45 \text { to } 0.62) \text {. } \\
\text { SE I.49 }(0.88 \text { to } 2.79) \text {. } \\
\text { SII I.29 (0.53 to } 2.32) \text {. } \\
\text { TST } 0.47 \text { ( }-0.27 \text { to I } .350) \text {. } \\
\text { Fatigue }-0.82 \text { (-I.87 to }-0.16) \text {. } \\
\text { Role functioning }-1.49 \text { (-I.78 to }-0.09) \text {. } \\
\text { Global QOL }-0.45 \text { (-I.3I to } 0.32) \text {. }\end{array}$ & $\begin{array}{l}\text { Sleep improved over baseline at weeks } 4 \text { and } 8 \text { and was related } \\
\text { to number of awakenings, WASO, SE, sleep quality, } \\
\text { and TST. } \\
\text { Fatigue improved by week } 8 \text {. } \\
\text { QOL role functioning scale improved from baseline, but not } \\
\text { statistically significant over time. }\end{array}$ & Low. \\
\hline $\begin{array}{l}\text { Pre-/post scores. } \\
\text { Global QOL }-1.09(-1.98 \text { to }-0.1 \mathrm{I}) \text {. } \\
\text { Cognitive } \mathrm{QOL}-0.54(-\mathrm{I} .4 \mathrm{I} \text { to } 0.370) \text {. } \\
\text { ISI } 2.67 \text { (I.37 to } 3.73) \text {. No data for TWT and SE. }\end{array}$ & $\begin{array}{l}\text { Eight treatments with cognitive behavioral intervention and education. } \\
\text { Treatment significantly improved sleep (ISI), sustained over time, } \\
\text { reduction of depressive symptoms and fatigue. Increase in global } \\
\text { and cognitive dimensions of } \mathrm{QOL} \text {, maintaining therapeutic gains } \\
\text { at } 3 \text { and } 6 \text { months. }\end{array}$ & Moderate. \\
\hline No reported means and SDs. & $\begin{array}{l}\text { Treatment group decreased hypnotic medication use, posttreatment } \\
\text { improved sleep efficiently in } 56 \% \text {, decreased anxiety and depression } \\
\text { and improved QOL (versus control), and } 12 \text { month follow-up showed } \\
\text { some enhancement. }\end{array}$ & Moderate/high. \\
\hline $\begin{array}{l}\text { QOL } 0.37(-0.11 \text { to } 0.83) \text {. } \\
\text { Insomnia }-0.37(0.84 \text { to } 0.10) \text {. }\end{array}$ & $\begin{array}{l}\text { Improved both groups, CBT improved fatigue, anxiety, depression, } \\
\text { with significant positive improvement in } \mathrm{QOL} \text {. } \\
\text { ISI severity correlated with } \mathrm{QOL}-0.39 \text { (significance } P=0.05 \text { ) posttreatment. }\end{array}$ & Moderate/high. \\
\hline $\begin{array}{l}\text { Posttreatment. } \\
\text { FACT-Physical } 0.58(0.19 \text { to } 0.97) \text {. } \\
\text { FACIT-F } 0.86(0.47 \text { to I.25). } \\
\text { Actigraph posttreatment TST }-0.8 \mathrm{I} \text { ( }(-\mathrm{I} .2 \mathrm{I} \text { to }-0.42) \text {. } \\
\text { SOL }-0.42(-0.80 \text { to }-0.0 \mathrm{I}) \text {. } \\
\text { WASO }-0.50(-0.89 \text { to }-0 . \mathrm{I}) \text {. }\end{array}$ & $\begin{array}{l}\text { CBT moderate effect in decreasing insomnia symptoms, increasing } \\
\text { physical and functional QOL, and reducing fatigue. }\end{array}$ & High. \\
\hline $\begin{array}{l}\text { Reported } d^{2} \text { posttreatment. } \\
\text { ISI }-1.29 . \\
\text { SOL }-0.88 . \\
\text { WASO }-1.35 . \\
\text { TST } 0.49 . \\
\text { SE\% I.19. } \\
\text { QLQ } 0.61 . \\
\text { DBAS total }-1.05 . \\
\end{array}$ & $\begin{array}{l}\text { From pre- to posttreatment, moderate-to-large effect size and } \\
\text { statistically and clinically significant differences on most sleep } \\
\text { variables and on QOL. } \\
\text { Dropout rate } 18 \% \text {. }\end{array}$ & Moderate. \\
\hline $\begin{array}{l}\text { Overall adjusted effect size: } \\
\text { ISI I.85. MFI total I.I6. HADS } 0.52 \text {. } \\
\text { SFI } 2 \text { mental } 0.48 \text {. SFI2 physical } 0.2 \text { I. } \\
\text { SE } 0.72 \text {. Soundness of sleep I.2I. } \\
\text { Restored sleep } 1.35 \text {. SOL } 0.67 \text {. }\end{array}$ & $\begin{array}{l}\text { Treatment group had reduced insomnia severity, increased sleep } \\
\text { efficiency, and no change in QOL scores. }\end{array}$ & Moderate. \\
\hline
\end{tabular}

Sleep disturbance.

Relax versus control 0.21 ( -0.104 to 0.52$)$. Autogenic versus control -0.25 ( -0.57 to 0.07$)$. EORTC QLQ-C30.

Relax versus control -0.08 ( -0.39 to 0.24$)$. Autogenic versus control 0.05 ( -0.27 to 0.37$)$.

\section{Pre-/posttreatment.}

Change in overall QOL $-0.50(-0.09$ to -0.54$)$.

No means (SDs) for Sleep Quality Improvement Statistic $X^{2}=6.8 \mathrm{I}, P<0.05$.
Significant improvements over time.

Low/moderate. Moderate or large effects on sleep latency, duration, efficiency, sleep quality, sleep medication, and daytime dysfunction. No difference between intervention groups.

QOL improved over time.

Significant improvements in overall QOL, SOSI, and sleep quality,

Moderate. but not correlated with dose.

QOL improvements associated with decreases in afternoon cortisol levels.

No changes in DHEAS and melatonin, but shifts in DHEAS

consistent with healthier profiles. 
Table 3 (Continued)

\begin{tabular}{|c|c|c|c|c|}
\hline Author, year & Intervention and design & Sample & Cancer types & Variables/measurement \\
\hline $\begin{array}{l}\text { Dhruva et al } \\
2012^{26}\end{array}$ & $\begin{array}{l}\text { Pranayama yoga breathing } \\
\text { techniques. } \\
\text { Pilot RCT crossover design. }\end{array}$ & $\begin{array}{l}\mathrm{N}=16 . \\
\mathrm{F}=14 . \\
\mathrm{M}=2 . \\
\text { Treatment }=8 . \\
\text { Control }=8 . \\
\text { Mean age } 52.4 \text { years. }\end{array}$ & $\begin{array}{l}\text { Breast, other. } \\
\text { Chemo treatment. }\end{array}$ & GSDS, MOS SF-I 2. \\
\hline $\begin{array}{l}\text { Chen et al, } \\
2013^{27} \text { (China) }\end{array}$ & $\begin{array}{l}\text { Qigong. } \\
\text { Movement, breathing, } \\
\text { meditation, and mindful focus } \\
\text { on the body. } \\
\text { RCT. }\end{array}$ & $\begin{array}{l}\mathrm{N}=96 . \\
\text { Treatment }=49 . \\
\text { Waitlist control }=47 . \\
\text { Mean age } 46 \text { years. }\end{array}$ & $\begin{array}{l}\text { Breast with radiation } \\
\text { treatment. }\end{array}$ & $\begin{array}{l}\text { PSQI, CES-D, BFI, FACT-G, cortisol } \\
\text { rhythm, saliva samples. }\end{array}$ \\
\hline $\begin{array}{l}\text { Milbury et al } \\
2013^{28}\end{array}$ & $\begin{array}{l}\text { TSM for cognitive dysfunction. } \\
\text { RCT 6-week versus waitlist } \\
\text { control. }\end{array}$ & $\begin{array}{l}\mathrm{N}=47 . \\
\text { Mean age } 56.3 \text { years. }\end{array}$ & $\begin{array}{l}\text { Breast with cognitive } \\
\text { impairment. }\end{array}$ & $\begin{array}{l}\text { PSQI, MOS SF36, FACT-Cog, FACT- } \\
\text { Spiritual, speed tasks. }\end{array}$ \\
\hline
\end{tabular}

\begin{tabular}{llll}
\hline Alvarez et al & EEG biofeedback to reduce & $\mathrm{N}=23$. & Breast. \\
$2013^{29}$ & cognitive impairment. & Mean age 56 years. & \\
& Prospective longitudinal & \\
& with waitlist control. &
\end{tabular}

\begin{tabular}{|c|c|c|c|c|}
\hline $\begin{array}{l}\text { Nakamura } \\
\text { et al } 2013^{30}\end{array}$ & $\begin{array}{l}\text { MBB MM interventions com } \\
\text { to SHE as active } \\
\text { control. } \\
\text { RCT. }\end{array}$ & $\begin{array}{l}\mathrm{N}=57 \\
\mathrm{~F}=43 . \\
\mathrm{M}=13 . \\
\mathrm{SHE}=19 . \\
\mathrm{MBB}=19 . \\
\mathrm{MM}=20 . \\
\text { Mean age } 53.5 \text { years. }\end{array}$ & $\begin{array}{l}\text { Cancer survivors with } \\
\text { sleep disturbance. } \\
\text { Breast and others. }\end{array}$ & $\begin{array}{l}\text { MOS medical sleep scale, FACT-G, } \\
\text { Perceived Stress Scale, CES-D, } \\
\text { WBI. }\end{array}$ \\
\hline $\begin{array}{l}\text { Wang et al } 20 \mathrm{II}^{3 \mathrm{I}} \\
\text { (Taiwan) }\end{array}$ & $\begin{array}{l}\text { Six-week walking program. } \\
\text { RCT. } \\
\text { Four time measures. }\end{array}$ & $\begin{array}{l}\mathrm{N}=72 . \\
\text { Treatment }=35 \\
\text { Control }=37 . \\
\text { Mean age } 5 \mathrm{I} . \mathrm{I} \text { years. }\end{array}$ & $\begin{array}{l}\text { Breast newly } \\
\text { diagnosed. }\end{array}$ & $\begin{array}{l}\text { PSQI, FACT-G, FACIT-F, } \\
\text { ESES, GLTEQ, 6MWT. }\end{array}$ \\
\hline
\end{tabular}

Abbreviations: 6MWT, 6-minute walk test; AT, autogenic training; BFI, Brief Fatigue Inventory; CBT, cognitive behavioral therapy; CES-D, Center for Epidemiologic Studies Depression Scale; Cl, confidence interval; d, Cohen's effect size; DBAS, Dysfunctional Beliefs and Attitudes About Sleep scale; DHEAS, dehydroepiandrosterone sulfate; EEG, electroencephalography; EORTC QLQ-C30, European Organisation for Research and Treatment of Cancer-Quality of Life Questionnaire; ESES, Exercise Self-Efficacy Scale; ESS, Epworth Sleepiness Scale; F, female; FACT, Functional Assessment of Cancer Therapy; FACT-B, Functional Assessment of Cancer Therapy-Breast; FACT-G, Functional Assessment of Cancer Therapy-General; FACT-Cog, Functional Assessment of Cancer Therapy-Cognitive; FACIT-F, Functional Assessment of Chronic IIIness Therapy-Fatigue; FSI, Fatigue Symptom Inventory; GI, gastrointestinal; GLTEQ, Godin Leisure-Time Exercise Questionnaire; GSDS, General Sleep Disturbance Scale; GYN, gynecological; HADS, hospital anxiety and depression scale; ISI, Insomnia Severity Index; M, male; MBB, mind-body bridging; MBSR, mindfulness-based stress reduction; MFI, multidimensional fatigue inventory; MM, mindfulness meditation; MOS, Medical Outcomes Study; MOS SF-I2, Medical Outcomes Study I2-Item Short-Form Health Survey; PR, progressive relaxation; PSG, polysomnography; PSQI, Pittsburgh Sleep Quality Index; QOL, quality of life; QLQ-C30, quality of life questionnaire; RCT, randomized controlled trial; SD, standard deviation; SE, sleep efficiency; SHE, sleep hygiene education; SII, Sleep Impairment Index; SOSI, Symptoms of Stress Inventory; SOL, sleep onset latency; TSM, Tibetan sound meditation; TST, total sleep time; TWT, total wake time; WASO, wake after sleep onset; WBI, World Health Organization Well-Being Index.

(by 56\%); this group also had decreased anxiety and depression and improved QOL. Measurement included rigorous subjective and objective sleep measures, although there were no reported means and standard deviations by which to calculate effect size. However, results indicated that the CBT-I approach to improving sleep also improved QOL and emotional distress. Savard et $\mathrm{al}^{20}$ more recently tested a 6-week self-help treatment for insomnia $(n=11$; no control group) using a sleep diary and other measures with moderate-to-large effect sizes pre-/posttreatment on most sleep variables and QOL, suggesting some benefit to using the self-help format; however, the $18 \%$ drop out rate is of some concern for applicability.

Ritterband et $\mathrm{al}^{21}$ tested an online CBT-I intervention $(n=28)$ with a waitlist control group and found that the treatment group had reduced insomnia and increased sleep efficiency, although there was no change in QOL; however, the low sample size limited the level of evidence. Dirksen 


\begin{tabular}{lll}
\hline Effect size $(\mathbf{C l})$ & Results & Quality score \\
\hline Sleep $0.86(-0.2 \mathrm{I}$ to 1.83$)$. & Increased dose of intervention in the number of hours practiced & Moderate. \\
QOL (mental score) $0.14(-0.85$ to I.I I). & resulted in improved sleep and QOL scores. & \\
QOL (Physical score) $-0.07(-1.04$ to 0.92$)$ & &
\end{tabular}

\begin{tabular}{|c|c|c|}
\hline 3 months posttreatment. & Qigong group had less depressive symptoms over time than control. & High. \\
\hline CES-D -0.20 (-0.60 to 0.20$)$. & Women with depressive symptoms at the beginning had less fatigue & \\
\hline BFI 0.17 ( -0.24 to 0.47$)$ & and better overall QOL. & \\
\hline PSQI 0.17 (0.24 to 0.57$)$ & Sleep scores improved, but not significantly. & \\
\hline FACT-G $-0.04(-0.44$ to 0.36$)$. & No significant improvement in cortisol slopes. & \\
\hline Post-program. & PSQI change from baseline effect size $=0.23$ and at I month $=0.32$. & Moderate. \\
\hline PSQI 0.32 (-0.3I to 0.94$)$. & TSM group demonstrated better short-term memory, & \\
\hline MOS SF36-mental 0.4 I (-0.22 to I.04). & cognitive abilities, and mental health spirituality at end of treatment & \\
\hline FACT-Cog 0.19 (-0.44 to $0.8 \mathrm{I})$. & but not I month later. & \\
\hline \multicolumn{3}{|l|}{ CES-D -0.1 I ( $(-0.73$ to 0.52$)$} \\
\hline \multicolumn{3}{|l|}{$\mathrm{BFI}-0.20(-0.82$ to 0.43$)$} \\
\hline Treatment versus normative sample. & Sleep scales (quality, daytime dysfunction, and global) were strongly & Moderate. \\
\hline FACT-Cog $-0.43(-0.92$ to 0.06$)$ & significantly improved. & \\
\hline FACIT-F 0.14 (-0.27 to 0.56$)$ & Two other sleep scales (latency and disturbance) were significantly & \\
\hline \multirow[t]{4}{*}{ PSQI I.I4 (0.6I to I.66). } & improved while use of sleep medication did not change. & \\
\hline & At follow-up, sample was similar to normative populations. & \\
\hline & Strong significant improvements on all four cognitive measures and & \\
\hline & impact on QOL, fatigue scale, and four psychological scales. & \\
\hline d. & Mean sleep disturbance symptoms in the MBB and MM groups & High. \\
\hline MOS sleep scale: MBB versus SHE I.06. & were lower than in the SHE group. & \\
\hline MM versus SHE 0.70 . & Also reported reductions in depression and improved mindfulness, & \\
\hline WBI: MBB versus SHE 0.52 . & self-compassion, and well-being. & \\
\hline
\end{tabular}

$M M$ versus SHE 0.28 .

\begin{tabular}{ll}
\hline Exercise versus usual care: & Exercise group had significantly better QOL, less fatigue, less sleep \\
FACT-G I.37 $(0.84$ to 1.86$)$. & disturbance, higher exercise self-efficacy, more exercise behavior, \\
FACIT-F I.2I $(0.69$ to I.70). & and greater exercise capacity than usual care group. \\
\hline PSQI $-0.13(-0.59$ to 0.33$)$. &
\end{tabular}

and Epstein 22 also tested 10-week CBT-I in an RCT with 72 breast cancer outpatients and found that treatment and control groups improved. The CBT group improved on fatigue, anxiety, depression, and QOL with low-to-moderate effect sizes. The CBT treatment group received a program with stimulus control, sleep restriction, and sleep education that included sleep hygiene, whereas the control groups received only sleep hygiene education. Improvement in both groups may have been a result of the sleep hygiene education. Espie et $\mathrm{al}^{23}$ more rigorously tested CBT-I in an RCT comprising a group of 150 breast and prostate cancer patients and a usualcare control group and found moderate effects in terms of decreased insomnia as well as increased physical and functional QOL. The study utilized both subjective and objective measurements of sleep with actigraphy, sleep diaries, and Epworth Sleepiness Scale.

Overall, the evidence suggests moderate-to-strong effects of CBT-I in reducing insomnia symptoms and improving 
QOL. Small sample sizes in $42 \%$ of the studies and lack of control groups in $42 \%$ of the studies limits the strength of the evidence for CBT-I.

\section{Mind-body interventions}

Eight studies included in this review involved mind-body interventions. Simeit et al, ${ }^{24}$ in a study of a German cancer rehabilitation program $(n=229)$, longitudinally tested two groups of cancer patients, implementing sleep management training (cognitive, sleep restriction, and stimulus control) with relaxation training. Patients were identified by the physicians as having sleep problems and self-selected progressive relaxation ( $\mathrm{n}=80)$ or autogenic training $(\mathrm{n}=71)$ with a control of 78 who had no training. There were significant improvements, with moderate-to-strong effects on sleep latency, duration, efficiency, sleep quality, and daytime dysfunction, with improvement in QOL over time. This study showed little difference between relaxation groups; however, improvements reflected the overall value of the rehabilitation program and patient preferences. The sample size was appropriate but lacked randomization.

Mind-body approaches have also been used during cancer treatment. Carlson et $\mathrm{al}^{25}$ tested a mindfulness-based stress reduction intervention in 69 breast and prostate cancer patients. Pre- and posttreatment changes were seen in overall QOL, stress, and sleep quality, but were not correlated with dose (hours of practice). This study also utilized a biologic measure of stress (cortisol), dehydroepiandrosterone sulfate, and melatonin; however, the only change was the change in the levels of dehydroepiandrosterone sulfate throughout the day and QOL improvements associated with decreases in afternoon cortisol levels. This study had no control group for comparison.

Another mind-body approach study was conducted by Dhruva et $\mathrm{al}^{26}$ in a pilot RCT crossover design study $(n=16)$ of pranayama yoga breathing techniques to improve chemotherapy-related symptoms. Increased dose, as measured by hours of practice, was associated with improvements in symptoms (fatigue, sleep disturbance, anxiety, depression, and stress) and mental QOL. The approach was feasible and showed effectiveness, but the small sample size influenced quality.

Chen et $\mathrm{al}^{27}$ conducted an RCT of Qigong, a mind-body practice, in 96 breast cancer patients with an average age of 45 years in China during radiation treatment and found less depressive symptoms over time than the control, less fatigue, and overall improved QOL. No difference in sleep was measured by PSQI and cortisol slopes, yet, unlike in other studies, comorbid insomnia was not an inclusion criterion for the study.
Mind-body techniques have been used in cancer survivors to improve symptoms. Milbury et $\mathrm{a}^{28}$ conducted an RCT of a 6-week program of Tibetan sound meditation $(n=47)$ for cognitive dysfunction and found improved memory, sleep (effect size [ES] 0.23 and at 1 month 0.32), and QOL. Another mind-body approach study by Alvarez et al ${ }^{29}$ prospectively followed 23 breast cancer patients with cognitive impairment who received electroencephalogram biofeedback to reduce cognitive impairment and were compared to a normative sample. Strong significant improvements on all cognitive measures impacted QOL, fatigue, and sleep scales (sleep quality, daytime dysfunction, global sleep, sleep latency and, sleep disturbance as measured by PSQI). The study by Alvarez et al was the only study in this category to report correlations among the subscales of sleep and impact on QOL; the significant results demonstrated that greater dysfunction in sleep quality correlated with poor performance in QOL.

Nakamura et $\mathrm{l}^{30}$ conducted an RCT $(\mathrm{n}=57)$ in cancer survivors with sleep disturbance to test the efficacy of a mind-body bridging and mindfulness meditation intervention compared to sleep hygiene education, and found that both mind-body bridging and mindfulness meditation led to diminished sleep-related symptoms than the sleep hygiene education group, as well as reduced depression and improved mindfulness and well-being.

These nonpharmacologic interventions involved different types of mind-body interventions, but they all involved patient engagement in relaxation or meditative practice, which show some promise for improving multiple symptoms related to QOL.

Exercise interventions have been used to improve sleep and QOL. Wang et $\mathrm{al}^{31}$ tested a 6-week walking program in Taiwan during treatment for breast cancer $(n=72)$ and demonstrated that increased physical activity decreased fatigue and sleep disturbances and improved QOL, although no correlations between sleep and QOL were reported.

Mind-body interventions demonstrated low-to-high effect sizes for sleep and QOL measures. The quality of the study designs was good overall, with $62 \%$ having a sample size $>50$ and $62 \%$ utilizing an RCT design.

In the overall summary of the 15 intervention studies that included outcome variables on sleep-wake disturbance and QOL, the issues of sample size ( $46 \%$ had below 50 subjects) and variety in settings and populations, cancer treatments and interventions, sleep and QOL measures, and study designs ( $40 \%$ lacked control group) overall limited the generalizability of the findings. However, effectiveness of the behavioral interventions on sleep and QOL demonstrated the 
Table 4 Sleep measurement scales used in review sample

\begin{tabular}{|c|c|c|}
\hline Sleep measurement scales & Studies using measure & Scoring and outcome measures \\
\hline $\begin{array}{l}\text { General Sleep Disturbance } \\
\text { Scale }\end{array}$ & Dhruva et al $2012^{26}$ & $\begin{array}{l}\text { Twenty-one items to evaluate various aspects of sleep disturbance. } \\
\text { Numeric rating scale } 0 \text { (never) to } 7 \text { (every day). } \\
\text { Total score = sum of seven subscale scores (quality of sleep, quantity } \\
\text { of sleep, sleep onset latency, mid-sleep awakenings, early awakenings, } \\
\text { medications for sleep, and excessive daytime sleepiness). } \\
\text { Zero (no disturbance) to I } 47 \text { (extreme sleep disturbance). }\end{array}$ \\
\hline Pittsburgh Sleep Quality Index & $\begin{array}{l}\text { Simeit et al } 2004 ;{ }^{24} \text { Espie et al } 2008 ; ;^{23} \\
\text { Beck et al } 2009 ; ;^{16} \text { Wang et al } 201 \mathrm{I} ; ;^{1} \\
\text { Alvarez et al } 2013 ;{ }^{29} \text { Chen et al } 2013 ;{ }^{27} \\
\text { Clevenger et al } 2013 ;{ }^{15} \text { Milbury et al } \\
2013 ; ;^{28} \text { Liu et al } 2013^{14}\end{array}$ & $\begin{array}{l}\text { Nineteen-item measure of sleep quality over the prior month. } \\
\text { Seven subscales: Latency, Duration, Efficiency, Disturbance, Use of Sleep } \\
\text { Medication, Daytime Sleep Dysfunction, and Subjective Sleep Quality. } \\
\text { Global sleep quality score range 0-2I with higher scores }(>5) \\
\text { indicating poor sleep quality. }\end{array}$ \\
\hline Insomnia Severity Index & $\begin{array}{l}\text { Quesnel et al } 2003 ;{ }^{18} \text { Savard et al } 2005 ;{ }^{19} \\
\text { Dirksen and Epstein } 2008 ; 22 \text { Savard et al } \\
2011 ;{ }^{20} \text { Ritterband et al } 2012^{21}\end{array}$ & $\begin{array}{l}\text { Seven-item scale evaluates perceived insomnia severity. } \\
\text { Total score range from 7-35. } \\
\text { Higher scores indicate greater insomnia. Clinical cut-off score is } 15 .\end{array}$ \\
\hline Epworth Sleepiness Scale & Espie et al $2008^{23}$ & $\begin{array}{l}\text { Likelihood of falling asleep when considering eight different daytime } \\
\text { activities. Range } 0-24 \text {. Score of }>8 \text { is excessive. } \\
\text { Assesses daytime sleepiness. }\end{array}$ \\
\hline Wrist actigraphy & Espie et al $2008,{ }^{23}$ Liu et al $2013^{14}$ & $\begin{array}{l}\text { Objective measure of motion and light recording continuously for } \\
\text { several days. } \\
\text { Total sleep time: hours during nocturnal sleep period. } \\
\text { Sleep latency: time to fall asleep prolonged if }>30 \text { minutes. } \\
\text { Sleep efficiency: ratio total sleep/time in bed ( } 85 \% \text { normal). } \\
\text { Wake after sleep onset: time awake after sleep onset. } \\
\text { Napping: period of } 10 \text { minutes of inactivity during day. } \\
\text { Circadian rhythm: sleep/rest patterns. }\end{array}$ \\
\hline
\end{tabular}

\begin{tabular}{ll}
\hline $\begin{array}{l}\text { European Organisation for } \\
\begin{array}{l}\text { Research and Treatment } \\
\text { of Cancer }\end{array}\end{array}$ & $\begin{array}{l}\text { Thirty questions under three headings: general state of well-being, } \\
\text { functional difficulties, and symptom control and perceived severity of } \\
\text { sleep problems. }\end{array}$ \\
& $\begin{array}{l}\text { Only includes one question about sleep: "Do you have trouble falling } \\
\text { asleep?" Answers are "not at all" =I, "a little" =2, "quite a bit" =3, } \\
\text { "very much" =4. }\end{array}$
\end{tabular}

\begin{tabular}{ll}
\hline Sleep diary & Davidson et al $2001 ;{ }^{17}$ Quesnel et al \\
& $2003 ; ;^{18}$ Savard et al $2005 ;{ }^{19}$ Espie et al \\
& $2008 ;{ }^{23}$ Savard et al $2011 ; ;^{20}$ Ritterband \\
& et al $2012^{21}$
\end{tabular}

Measures self-reported time in bed, sleep efficiency, sleep latency, wake after sleep onset, and quality of sleep.

Sleep Impairment Index

Davidson et al $2001^{17}$

A self-report instrument that elicits the subject's perception of the level of severity, distress, and impairment of daytime functioning associated with insomnia.

Items assessed: severity of sleep latency, sleep maintenance, early morning awakenings, satisfaction with current sleep patterns, interference with daily functioning, noticeable impairment, concern about sleep problem, contributing factors, and outcomes.

\begin{tabular}{lll}
\hline Polysomnography & $\begin{array}{l}\text { Quesnel et al } 2003 ;{ }^{18} \text { Savard et al } \\
2005 ;{ }^{19} \text { Clevenger et al } 2013^{15}\end{array}$ & $\begin{array}{l}\text { Objective study of sleep requiring an in-lab overnight sleep. Measures } \\
\text { sleep stages, apneas, oxygen saturation, and other physiologic variables. }\end{array}$ \\
\hline $\begin{array}{l}\text { Medical Outcomes Study Sleep } \\
\text { Scale (MOS-SS) }\end{array}$ & $\begin{array}{l}\text { Nakamura et al } 2013^{30} \\
\text { Consisting of I } 2 \text { items, the sleep scale is only a small part of the } \\
\text { complete Patient Assessment Questionnaire, a 20-page instrument } \\
\text { querying a broad range of health-related issues including physical } \\
\text { functioning, psychological well-being, health distress, and pain. } \\
\text { The sleep scale examines six factors: sleep initiation, maintenance, } \\
\text { respiratory problems, quantity, perceived adequacy, and somnolence. }\end{array}$
\end{tabular}

usefulness of these approaches in patients with cancer. While most of these studies did not report correlations between sleep and QOL, they did demonstrate some effectiveness in improving sleep and QOL outcomes.

\section{Measurement of sleep-wake disturbance and QOL}

Historically, measurement of sleep-wake disturbance was a single question on a symptom and QOL scales that 
Table 5 Quality of life (QOL) measures used in the reviewed studies

\begin{tabular}{|c|c|c|}
\hline QOL measures & Studies using measure & Scoring \\
\hline $\begin{array}{l}\text { Functional Assessment of } \\
\text { Cancer Therapy } \\
\text { (FACT)-General } \\
\text { Subscales: } \\
\text { FACT-Breast } \\
\text { FACT-Ovarian } \\
\text { FACT-Cognitive } \\
\text { FACT-Functional }\end{array}$ & $\begin{array}{l}\text { General: Espie et al } 2008 ; ;^{23} \text { Wang et al } 2011 ; ;^{31} \\
\text { Nakamura et al } 20133^{30} \text { Chen et al } 2013^{27} \\
\text { Subscales: } \\
\text { FACT-Breast: Dirksen and Epstein } 2008^{22} \\
\text { FACT-Ovarian: Clevenger et al } 2013^{15} \\
\text { FACT-Cognitive: Alvarez et al } 2013 ; 29 \text { Milbury } \\
\text { et al } 2013^{28} \\
\text { FACIT-Fatigue: Alvarez et al } 2013^{29}\end{array}$ & $\begin{array}{l}\text { Assesses multiple dimensions of QOL - physical, } \\
\text { emotional, social, and functional well-being; } \\
\text { relationship with physician; and 9-item general } \\
\text { measure of health-related QOL. Five-point scale } \\
\text { from } 0 \text { (not at all) to } 4 \text { (very much). Scale scores } \\
\text { added to obtain total score. } \\
\text { Subscales to address associated symptoms: } \\
\text { Breast } \\
\text { Ovarian } \\
\text { Cognitive } \\
\text { Fatigue. }\end{array}$ \\
\hline $\begin{array}{l}\text { European Organization Organisation for } \\
\text { Research and Treatment of } \\
\text { Cancer-Quality of Life } \\
\text { Questionnaire }\end{array}$ & $\begin{array}{l}\text { Carlson et al } 2004 ; ;^{25} \text { Simeit et al } 2004 ; ;^{24} \\
\text { Savard et al } 2005 ;{ }^{19} \text { Savard et al } 20 \mathrm{I} \mathrm{I}^{20}\end{array}$ & $\begin{array}{l}\text { Thirty questions under three headings: general } \\
\text { state of well-being, functional difficulties, symptom } \\
\text { control. Subscale-global QOL: each item rated on } \\
\text { a 4-point Likert scale. Scale of I to } 7 \text { (very bad to } \\
\text { excellent). } \\
\text { Evaluation of general QOL. }\end{array}$ \\
\hline Quality of Life-Cancer & Quesnel et al $2003^{18}$ & $\begin{array}{l}\text { Thirty-item measure that uses } 100 \mathrm{~mm} \text { linear } \\
\text { analog scale for response. From } 0-100 \text {. Subscales } \\
\text { included physical, cognitive, social and role function. }\end{array}$ \\
\hline $\begin{array}{l}\text { Medical Outcomes Survey-Health } \\
\text { Related Quality of Life - } \\
\text { physical (PCS) and mental (MCS) } \\
\text { composite scales of Short Form- } \\
36 \text { version } 2 \text { MOS SF-I } 2\end{array}$ & $\begin{array}{l}\text { Milbury et al } 2013^{28} \\
\text { Beck et al } 2009 ;{ }^{16} \text { Dhruva et al } 2012 ;{ }^{26} \\
\text { Ritterband et al } 2012^{21}\end{array}$ & $\begin{array}{l}\text { Health-related QOL measure, } 36 \text {-question survey. } \\
\text { Eight health domains divided into PCS and MCS } \\
\text { scores. PCS and MCS mean scores and standard } \\
\text { deviations ranged from } 0 \text { to } 100 \text {, with higher } \\
\text { scores indicating better health and well-being. } \\
\text { Mean } \pm \text { standard deviation }=50 \pm 10 \text { for the US } \\
\text { general population. }\end{array}$ \\
\hline
\end{tabular}

Abbreviations: PCS, physical component score; MCS, mental component score; MOS-SF-12, Medical Outcomes Study I2-Item Short-Form Health Survey.

asked "Do you have trouble sleeping?"; however, as the complexity of sleep disturbance became known, ${ }^{3,12}$ more comprehensive subjective and objective sleep measures were utilized, as delineated in Table 4. Using both subjective and objective measures is helpful in measuring sleep disturbance, since perceived sleep may not correlate with the actual sleep parameters. Only a few studies ${ }^{25,27}$ used biologic measures that added insight into the physiological mechanisms, inflammatory responses, and the role of stress influencing sleep. Berger et $\mathrm{al}^{3}$ recommend nine parameters to fully assess sleep: total sleep time; sleep latency; awakenings; wake after sleep onset; napping; sleepiness in daytime; perceived sleep; circadian rhythms; and sleep efficiency.

The assessment of QOL measurements as outlined in Table 5 shows that multiple dimensions such as health-related, social, and psychological functions related to well-being were used in the studies. Most of the QOL measures have been validated in the cancer literature and show good reliability and validity. Cancer-specific QOL tools were used in 78\% of the studies, including the Functional Assessment of Cancer
Therapy (FACT), which was used in 50\% of the studies, and FACT gives consideration to treatment and disease-related symptoms.

\section{Discussion}

After synthesizing findings from this narrative review, the evidence suggests an interrelationship between increasing sleep-wake disturbances and poorer QOL in cancer patients. Only $2 \%$ of the identified literature met the parameters for inclusion, illustrating the dearth of studies that included both sleep-wake disturbances and QOL. While the majority of studies suggested that poor sleep was associated with poorer QOL, only $30 \%$ of the reviewed studies reported the actual correlations between sleep and QOL. The majority of the studies involved women with breast cancer, which limits applicability to men and other cancer types. In addition, the studies were heterogeneous, with varied study designs, sample sizes, settings, cancer types and stages, and types of treatment, which limits generalizability.

This review provides a comprehensive assessment of QOL; however, the evaluation of sleep-wake disturbances 


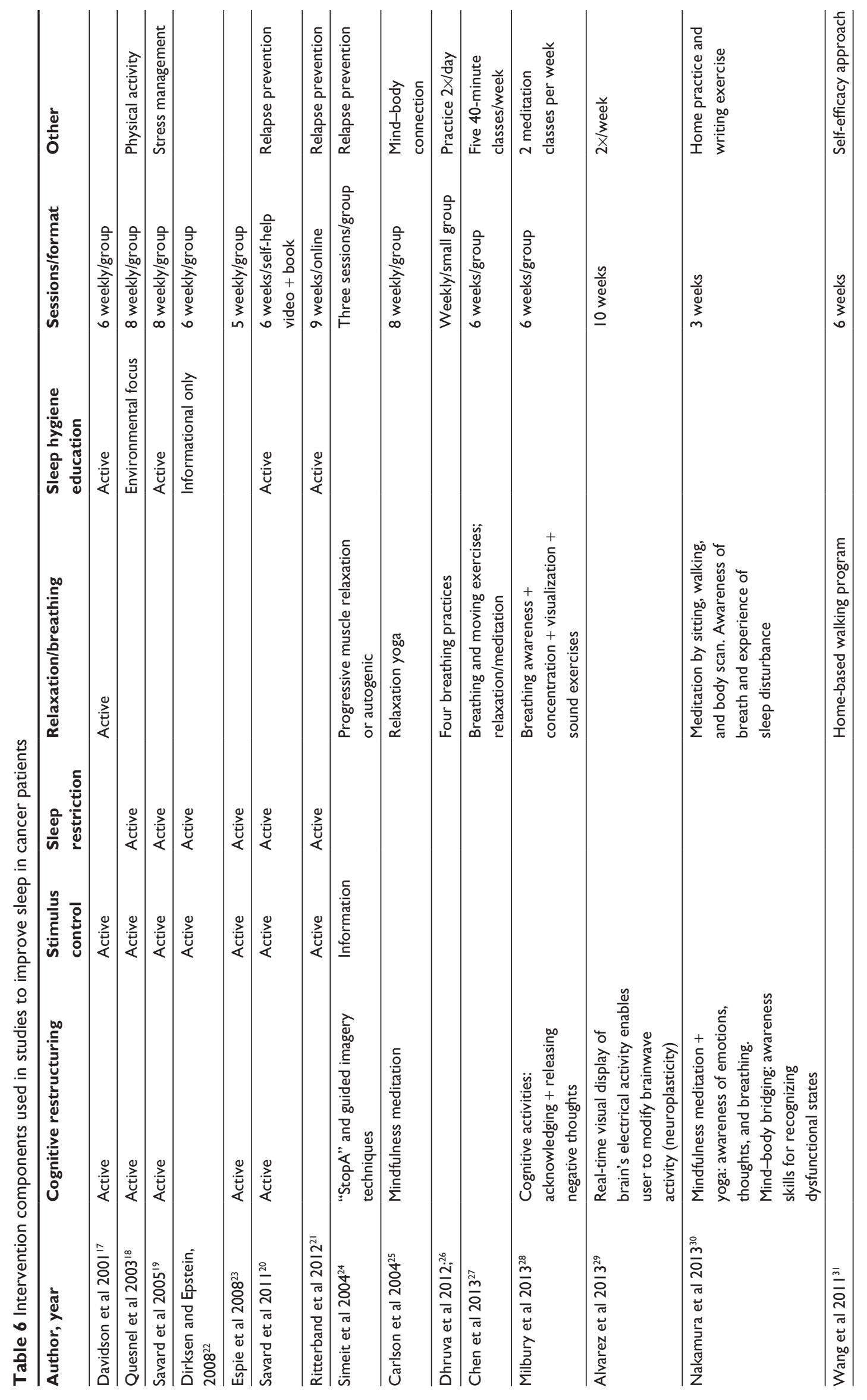


varied among studies and sometimes included subjective and objective measures as outlined in Tables 4 and 5 . In addition, researchers identified multifactorial components influencing sleep-wake disturbances, including anxiety, depression, pain, and fatigue, which have been shown to occur in clusters. . $^{6,32}$ Nonetheless, QOL measures offer a way to encompass a more holistic perspective of the outcomes of sleep-wake disturbance that includes social, mental, emotional, and physical measures.

The reviewed interventions demonstrate feasible approaches to improving sleep-wake disturbance in cancer patients that result in improved QOL outcomes, but the evidence is not enough to make a strong recommendation (see Table 6 for a summary of intervention components). In standard CBT-I, five elements are included, ie, cognitive restructuring, stimulus control, sleep restriction, relaxation, and sleep hygiene education. Most CBT-I interventions in the reviewed studies included stimulus control and sleep restriction; however, relaxation was only used in one study. The reviewed studies showed moderate-to-strong effect sizes in reducing insomnia symptoms and improving QOL, although the quality ratings given to the studies ranged from low to high, limiting recommendation. Mind-body interventions included behavioral components such as relaxation/breathing, mindfulness meditation, and movements such as walking and yoga. These studies demonstrate varied effects (from low to high) on improving sleep and QOL, and were given quality ratings ranging from low to high. Similarities between CBT-I and mind-body interventions included behavioral and cognitive processes to reduce stress.

In this review, it was noted that cancer patients' prolonged daytime napping patterns and shorter nighttime sleep negatively influenced QOL, suggesting congruence with the two-process model, as suggested by Vena et al, ${ }^{11}$ in evaluating sleep-wake disturbance. The CBT-I component of sleep restriction and sleep hygiene offered some support in reducing napping, supporting the development of sleep pressure, and consolidating sleep at night. The model also infers the importance of circadian rhythms in promoting good sleep. Relatively unstudied is the implication that disrupted circadian rhythms result in decrements in QOL and mortality. It is important to monitor circadian rhythms during treatment, since patients are often encouraged to nap as a result of side effects and increasing fatigue, ${ }^{33}$ which may ultimately weaken their circadian rhythms and homeostatic sleep drives. This was also evident when patients reported having their nights and days mixed up (Dickerson, unpublished data, 2014). Actigraphy and sleep diaries are effective instruments for measuring this effect. A study tested interventions that strengthen circadian rhythm function are beginning to demonstrate the protective effect of treatments such as bright light therapy during chemotherapy in patients with breast cancer. ${ }^{34}$ In addition, implementation of future basic sleep hygiene education in patients with cancer regarding the benefits of avoiding excessive daytime napping ( $>30$ minutes) may improve sleep and QOL; however, this requires further validation research in cancer patients.

\section{Implications for the practice setting}

From this review, it is evident that sleep disturbance in patients with cancer is problematic and affects their QOL. Cognitive behavioral interventions showed some moderate effectiveness on improving sleep, as did mind-body interventions. Based on this narrative review, the recommendations for practice include:

1. Providers routinely including sleep assessments ${ }^{35}$ as part of the care for patients with cancer, thereby increasing awareness of sleep patterns and potential for disruptions.

2. Providers teaching basic sleep hygiene as a standard of care and advising cancer patients (in treatment) to avoid excessive napping ( $>30$ minutes) to avoid circadian rhythm disruption and promote consolidated sleep at night.

3. Prescribing CBT-I or mind-body approaches for patients with insomnia.

Since sleep is often not a priority for patients dealing with cancer, ${ }^{2}$ the onus is on the providers to institute the recommendations into routine clinical practice/care.

\section{Future research}

Given the prevalence of sleep-wake disturbance in cancer patients, this review demonstrates the need for research studies to include sleep and QOL instruments that comprehensively assess sleep quality versus the use of a single-item assessment. Use of the subjective and objective measurement tools expands the knowledge of the influence of sleep on QOL. Future research endeavors should also explore the role of circadian rhythms in sleep-wake disturbances, which will facilitate understanding of the effect of sleep disturbance on the multiple components of QOL. Future research should extend the work on the interventions reviewed herein and determine whether these interventions are sustainable and applicable to other cancer diagnoses. Since breast cancer was the main diagnosis in the studies (in eight of the 15 studies), males have been underrepresented. Additionally, 
intervention studies must be expanded to other cancer diagnostic groups and replicated in larger samples. Overall recommendations are: to establish standard instruments for QOL and sleep that could be used in clinical trials and practice to measure sleep and facilitate meta-studies; to expand the study of sleep and QOL to more populations and ethnic groups; and to further test the interventions in larger trials to improve the level of evidence.

\section{Limitations of this review}

In this review, the research team reviewed and cross-validated the results and synthesized the data. However, it is possible that some studies were overlooked by, for example, limiting the search to English-language databases and to studies that had both sleep disturbance and QOL measures. The overall level of evidence was determined to be moderate.

\section{Conclusion}

Poor sleep negatively impacts quality of life, as evidenced in the studies included in this review. The intervention studies included nonpharmacologic interventions such as cognitive behavioral treatment and mind-body and exercise interventions, with moderate-to-high levels of evidence for improvement in sleep measures and QOL.

\section{Disclosure}

The authors report no conflicts of interest in this work.

\section{References}

1. Moher D, Liberati A, Tetzlaff J, Altman DG; P RISMA Group. Preferred reporting items for systematic reviews and meta-analyses: the PRISMA statement. Ann Intern Med. 2009;151:264-269, W64.

2. Dickerson SS, Sabbah EA, Ziegler P, Chen H, Steinbrenner LM, Dean G. The experience of a diagnosis of advanced lung cancer: sleep is not a priority when living my life. Oncol Nurs Forum. 2012;39:492-499.

3. Berger AM, Parker KP, Young-McCaughan S, et al. Sleep wake disturbances in people with cancer and their caregivers: state of the science. Oncol Nurs Forum. 2005;32:E98-E126.

4. Clark J, Cunningham M, McMillan S, Vena C, Parker K. Sleep-wake disturbances in people with cancer part II: evaluating the evidence for clinical decision making. Oncol Nurs Forum. 2004;31:747-771.

5. Ancoli-Israel S, Moore PJ, Jones V. The relationship between fatigue and sleep in cancer patients: a review. Eur J Cancer Care (Engl). 2001;10:245-255.

6. Dodd MJ, Miaskowski C, Paul SM. Symptom clusters and their effect on the functional status of patients with cancer. Oncol Nurs Forum. 2001;28:465-470.

7. Redeker NS, Lev EL, Ruggiero J. Insomnia, fatigue, anxiety, depression, and quality of life of cancer patients undergoing chemotherapy. Sch Inq Nurs Pract. 2000;14:275-290.

8. Dodd MJ, Cho MH, Cooper BA, Miaskowski C. The effect of symptom clusters on functional status and quality of life in women with breast cancer. Eur J Oncol Nurs. 2010;14:101-110.

9. Dodd MJ, Cho MH, Cooper BA, et al. Identification of latent classes in patients who are receiving biotherapy based on symptom experience and its effect on functional status and quality of life. Oncol Nurs Forum. 2011;38:33-42.
10. Miaskowski C, Cooper B, Paul SM, et al. Identification of patient subgroups and risk factors for persistent breast pain following breast cancer surgery. J Pain. 2012;13:1172-1187.

11. Vena C, Parker K, Cunningham M, Clark J, McMillan S. Sleep-wake disturbances in people with cancer part I: an overview of sleep, sleep regulation, and effects of disease and treatment. Oncol Nurs Forum. 2004;31:735-746.

12. Berger AM, Sankaranarayanan J, Watanabe-Galloway S. Current methodological approaches to the study of sleep disturbances and quality of life in adults with cancer: a systematic review. Psychooncology. 2007;16:401-420.

13. Borbély AA. A two process model of sleep regulation. Hum Neurobiol. 1982;1:195-204.

14. Liu L, Fiorentino L, Rissling M, et al. Decreased health-related quality of life in women with breast cancer is associated with poor sleep. Behav Sleep Med. 2013;11:189-206.

15. Clevenger L, Schrepf A, Degeest K, et al. Sleep disturbance, distress, and quality of life in ovarian cancer patients during the first year after diagnosis. Cancer. 2013;119:3234-3241.

16. Beck SL, Towsley GL, Caserta MS, Lindau K, Dudley WN. Symptom experiences and quality of life of rural and urban older adult cancer survivors. Cancer Nurs. 2009;32:359-369.

17. Davidson JR, Waisberg JL, Brundage MD, MacLean AW. Nonpharmacologic group treatment of insomnia: a preliminary study with cancer survivors. Psychooncology. 2001;10:389-397.

18. Quesnel C, Savard J, Simard S, Ivers H, Morin CM. Efficacy of cognitive-behavioral therapy for insomnia in women treated for nonmetastatic breast cancer. J Consult Clin Psychol. 2003;71:189-200.

19. Savard J, Simard S, Ivers H, Morin CM. Randomized study on the efficacy of cognitive-behavioral therapy for insomnia secondary to breast cancer, part I: sleep and psychological effects. J Clin Oncol. 2005;23:6083-6096.

20. Savard J, Villa J, Simard S, Ivers H, Morin CM. Feasibility of a selfhelp treatment for insomnia comorbid with cancer. Psychooncology. 2011;20:1013-1019.

21. Ritterband LM, Bailey ET, Thorndike FP, Lord HR, Farrell-Carnahan L, Baum LD. Initial evaluation of an Internet intervention to improve the sleep of cancer survivors with insomnia. Psychooncology. 2012;21:695-705.

22. Dirksen SR, Epstein DR. Efficacy of an insomnia intervention on fatigue, mood and quality of life in breast cancer survivors. JAdv Nurs. 2008;61:664-675.

23. Espie CA, Fleming L, Cassidy J, et al. Randomized controlled clinical effectiveness trial of cognitive behavior therapy compared with treatment as usual for persistent insomnia in patients with cancer. J Clin Oncol. 2008;26:4651-4658.

24. Simeit R, Deck R, Conta-Marx B. Sleep management training for cancer patients with insomnia. Support Care Cancer. 2004; 12:176-183.

25. Carlson LE, Speca M, Patel KD, Goodey E. Mindfulness-based stress reduction in relation to quality of life, mood, symptoms of stress and levels of cortisol, dehydroepiandrosterone sulfate (DHEAS) and melatonin in breast and prostate cancer outpatients. Psychoneuroendocrinology. 2004;29:448-474.

26. Dhruva A, Miaskowski C, Abrams D, et al. Yoga breathing for cancer chemotherapy-associated symptoms and quality of life: results of a pilot randomized controlled trial. J Altern Complement Med. 2012;18: 473-479.

27. Chen Z, Meng Z, Milbury K, et al. Qigong improves quality of life in women undergoing radiotherapy for breast cancer: results of a randomized controlled trial. Cancer. 2013;119:1690-1698.

28. Milbury K, Chaoul A, Biegler K, et al. Tibetan sound meditation for cognitive dysfunction: results of a randomized controlled pilot trial. Psychooncology. Epub May 9, 2013.

29. Alvarez J, Meyer FL, Granoff DL, Lundy A. The effect of EEG biofeedback on reducing postcancer cognitive impairment. Integr Cancer Ther. 2013;12:475-487. 
30. Nakamura Y, Lipschitz DL, Kuhn R, Kinney AY, Donaldson GW. Investigating efficacy of two brief mind-body intervention programs for managing sleep disturbance in cancer survivors: a pilot randomized controlled trial. J Cancer Surviv. 2013;7:165-182.

31. Wang YJ, Boehmke M, Wu YW, Dickerson SS, Fisher N. Effects of a 6-week walking program on Taiwanese women newly diagnosed with early-stage breast cancer. Cancer Nurs. 2011;34:E1-E13.

32. Miaskowski C, Cooper BA, Paul SM, et al. Subgroups of patients with cancer with different symptom experiences and quality-of-life outcomes: a cluster analysis. Oncol Nurs Forum. 2006;33:E79-E89.
33. Fernandes R, Stone P, Andrews P, Morgan R, Sharma S. Comparison between fatigue, sleep disturbance, and circadian rhythm in cancer inpatients and healthy volunteers: evaluation of diagnostic criteria for cancer-related fatigue. J Pain Symptom Manage. 2006;32:245-254.

34. Neikrug AB, Rissling M, Trofimenko V, et al. Bright light therapy protects women from circadian rhythm desynchronization during chemotherapy for breast cancer. Behav Sleep Med. 2012;10:202-216.

35. Lee KA, Ward TM. Critical components of a sleep assessment for clinical practice settings. Issues Ment Health Nurs. 2005;26:739-750.

\section{Publish your work in this journal}

Nature and Science of Sleep is an international, peer-reviewed, open access journal covering all aspects of sleep science and sleep medicine, including the neurophysiology and functions of sleep, the genetics of sleep, sleep and society, biological rhythms, dreaming, sleep disorders and therapy, and strategies to optimize healthy sleep. The journal welcomes

\section{Dovepress}

original research, clinical \& epidemiological studies, reviews \& evaluations, case reports and extended reports. The manuscript management system is completely online and includes a very quick and fair peerreview system, which is all easy to use. Visit http://www.dovepress.com/ testimonials.php to read real quotes from published authors. 